06

\title{
Фоторазрушение субмикронных кристаллов DAST в пленке PMMA путем прямой лазерной записи
}

\author{
() Т.Н. Погосян ${ }^{1,2}$, И. Ю. Денисюк ${ }^{1, \uparrow}$, Н.Д. Лай ${ }^{2}$, И. Леду-Рак ${ }^{2}$ \\ ${ }^{1}$ Университет ИТМО, \\ 197101 Санкт-Петербург, Россия \\ ${ }^{2}$ ENS Paris-Saclay, \\ 94230 Cachan, France \\ ฯ e-mail: denisiuk@mail.ifmo.ru
}

Поступила в редакцию 24.12.2018 г.

В окончательной редакции 20.06.2019 г.

Принята к публикации 20.06.2019 г.

\begin{abstract}
Исследованы процессы избирательного фотовыцветания нелинейных нанокристаллов DAST (trans-4'-(dimethylamino)-Nmethyl-4-stilbazolium tosylate) в пленке из полиметилметакрилата толщиной $2 \mu \mathrm{m}$ с помощью прямой лазерной записи на длине волны $532 \mathrm{~nm}$ при мощности $1-10 \mathrm{~mW}$ и скорости записи 5-35 $\mathrm{m} / \mathrm{s}$. Выявлена зависимость вида записанной картины от параметров записи. В результате разрушения кристаллов и перехода их в аморфную форму записана структура, которая хорошо различима в оптический микроскоп и при сканировании сигнала люминесценции. Нарушение поверхности слоя в местах разрушения кристаллов не выявлено. Метод позволит формировать решетки с периодической модуляцией нелинейно-оптического коэффициента, что важно для преобразования в режиме квазифазового синхронизма.
\end{abstract}

Ключевые слова: фотовыцветание, нелинейная оптика, DAST, 2D решетки.

DOI: $10.21883 /$ OS.2019.10.48376.372-18

\section{Введение}

Работа посвящена исследованию записи двумерной (2D) решетки в нанокомпозитах полиметилметакрилата (Poly(methyl methacrylate), PMMA) с субмикронными нелинейными кристаллами DAST (trans4'-(dimethylamino)-Nmethyl-4-stilbazolium tosylate), основанной на фоторазрушении кристаллов излучением лазера высокой интенсивности. Цель формирования периодических решеток в нелинейно-оптическом материале генерация гармоник [1,2], оптическая параметрическая генерация [3], нелинейное формирование луча заданной формы [4-6] и другие нелинейно-оптические эффекты, усиленные методом фазовой квазисинхронизации (ФКС), что обеспечивает высокую эффективность преобразования.

Молекулярный кристалл DAST обладает одним из наибольших нелинейных оптических коэффициентов среди известных материалов. Исследование нелинейнооптических свойств монокристаллических слоев толщиной 0.1-1 mm на телекоммуникационных длинах волн показало, что коэффициенты нелинейности равны $d_{111}=1010 \mathrm{pm} / \mathrm{V}, d_{122}=96 \mathrm{pm} / \mathrm{V}$ и $d_{212}=53 \mathrm{pm} / \mathrm{V}$ на длине волны $1.318 \mu \mathrm{m}, d_{111}=290 \mathrm{pm} / \mathrm{V}, d_{122}=41 \mathrm{pm} / \mathrm{V}$ и $d_{212}=39 \mathrm{pm} / \mathrm{V}$ на $1.55 \mu \mathrm{m}[7,8]$. По этим причинам DAST был выбран в качестве объекта исследования. Метод создания нелинейных решеток основывался на избирательном фоторазрушении областей субмикронных кристаллов в нанокомпозите, что позволило получить не только 2D структуры с ФКС в тонких пленках, но и в трехмерные (3D) структуры с ФКС в толстых пленках для экспериментальной реализации работы [9]. Для фоторазрушения использовалась установка, описанная в работе [10]. Длина волны $532 \mathrm{~nm}$ близка к максимуму поглощения пленки РММА с кристаллами DAST, поэтому на ней возможна лишь 2D запись, для 3D записи потребуется использование двухфотонного поглощения, например, мощного излучения с длиной волны $1064 \mathrm{~nm}$.

\section{Материалы и методы}

В работе использовались пленки РММА с субмикронными кристаллами DAST, полученные путем зародышеобразования молекулярного кристалла в полимерной матрице, как описано в работе [11]. Используемые компоненты: PMMA (Plexiglas ${ }^{\circledR}$ V045) и DAST (CAS: 80969-52-4 Genolite biotek). В качестве растворителей использованы хлороформ (CL0218 Scharlau) для PMMA (концентрация 5\%) и метанол (322415 Aldrich) для DAST, концентрация которого соответствовала пределу растворимости при комнатной температуре [12]. Растворы смешаны в соотношении, при котором доля DAST составила 2\% от массы РММА. Фазовое разделение на PMMA в хлороформе и раствор DAST в метаноле (микропузырьки) происходит при получении пленки на центрифуге: первая ступень $100 \mathrm{rpm}$ и вторая ступень $1500 \mathrm{rpm}$ в течение $10 \mathrm{~s}$. При быстром испарении растворителя в каждом пузырьке образуются субмикронные частицы DAST. На рис. 1 представлено изображение кристаллов DAST со средним размером $300 \mathrm{~nm}$, 


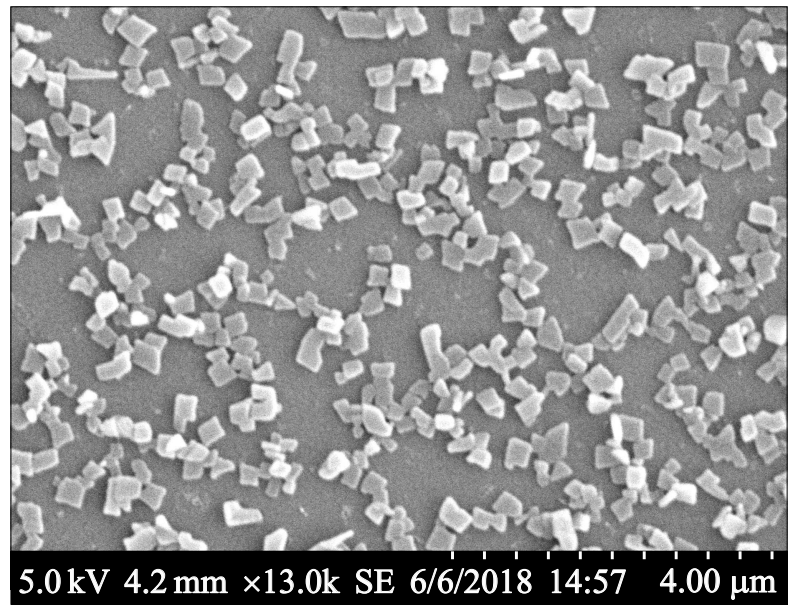

Рис. 1. SEM-изображение кристаллов DAST.

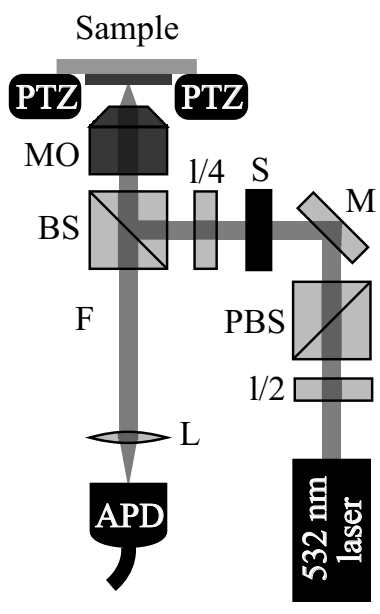

Pис. 2. Схема установки для прямой лазерной записи и сканирования сигнала люминесценции: РZT - пьезоэлектрический привод, МО - микрообъектив, $\lambda / 4$ - четвертьволновая пластинка, $\lambda / 2-$ полуволновая пластинка, BS - светоделительная пластина, PBS - поляризационная светоделительная пластина, $\mathrm{M}$ - зеркало, $\mathrm{S}$ - электронный затвор, L - линза, $\mathrm{APD}$ - лавинный фотодиод.

полученное с помощью сканирующего электронного микроскопа SEM Hitachi S-3400N. Отжиг пленки при температуре $180^{\circ} \mathrm{C}$ в течение $30 \mathrm{~min}$ способствует выходу растворителя и преобразованию аморфного DAST в кристаллическую форму [11]. Толщина используемой в эксперименте пленки равна $2 \mu \mathrm{m}$.

На рис. 2 представлена схема установки для прямой лазерной записи и сканирования сигнала люминесценции. В установке используется лазер Oxxius $\mathrm{Nd}-\mathrm{YAG}$ с преобразованием излучения во вторую гармонику, рабочая длина волны - $532 \mathrm{~nm}$, максимальная мощность $300 \mathrm{~mW}$, длина когерентности - $300 \mathrm{~m}$; стабильность наведения луча $-0.005 \mathrm{mrad} /{ }^{\circ} \mathrm{C}$. Для изменения мощности лазерного излучения используются полуволновая и поляризационная пластинки. Сигнал люминесценции детектируется лавинным фотодиодом SPCM-AQRH-13 (Perkin Elmer). Пьезоэлектрический привод позволяет с большой точностью перемещать образец относительно неподвижного сфокусированного лазерного луча. Благодаря этому и электронному затвору возможна поточечная запись любого заданного изображения. Параметры записывающей области зависят от мощности излучения и от особенностей материала. Область имеет форму вытянутого сфероида. Диаметр поперечного сечения не превышает $1 \mu \mathrm{m}$. На образцах записывалась двумерная решетка $5 \times 5$ линий с периодом $15 \mu \mathrm{m}$. В ходе эксперимента выбиралась скорость записи от 5 до $35 \mu \mathrm{m} / \mathrm{s}$ при мощности лазерного пучка 1,5 или $10 \mathrm{~mW}$.

Записанные изображения сканировались лазерным лучом без изменения положения образца, чтобы получить картину сигнала люминесценции кристаллов DAST, a затем анализировались на оптическом микроскопе Nicon Eclipse $\mathrm{Ci}-\mathrm{S}$.

\section{Результаты и обсуждение}

В результате воздействия на нанокомпозит лазерного излучения наблюдалось исчезновение люминесценции DAST без образования видимых повреждений слоя. Молекулярный кристалл DAST при нагреве и быстром охлаждении переходит в аморфную форму, люминесценция которой имеет квантовый выход $0.2 \%$, много меньше в сравнении с кристаллической формой, квантовый выход люминесценции которой 14-20\%. По этим причинам мы считаем, что лазерная запись на нанокомпозите основана на термически индуцированном преобразовании DAST из кристаллической в аморфную форму.

Эксперимент показал, что в ходе медленной $(5 \mu \mathrm{m} / \mathrm{s})$ записи при мощности $1 \mathrm{~mW}$ (рис. 3,a) термическое влияние поглощенного излучения разрушает окружающие кристаллы, в результате не наблюдается четкой записи. Увеличение мощности до $5 \mathrm{~mW}$ позволяет более четко сформировать заданное изображение, однако сохраняется ореол термического влияния на окружающие кристаллы (рис. $3, b$ ). Если увеличить скорость записи в два раза - до $10 \mu \mathrm{m} / \mathrm{s}$ (рис. 3,c), то качество записи структуры заметно улучшается.

Полученные результаты указывают на преимущество записи при высокой мощности и высокой скорости. На рис. 3, $d$ представлено люминесцентное изображение картины, записанной при мощности $10 \mathrm{~mW}$ и скорости $35 \mu \mathrm{m} / \mathrm{s}$. Ширина записанной линии не превышает $1 \mu \mathrm{m}$, что связано с высоким поглощением лазерного излучения материалом и с размером самих кристаллов DAST. В широкопольный оптический микроскоп отчетливо видна записанная структура и линии разрушенных кристаллов (рис. 3,e-f). Изучение поверхности на профилометре Dektak 150 не выявило никаких изменений рельефа образца, РММА не был затронут термическим влиянием. 
$a$

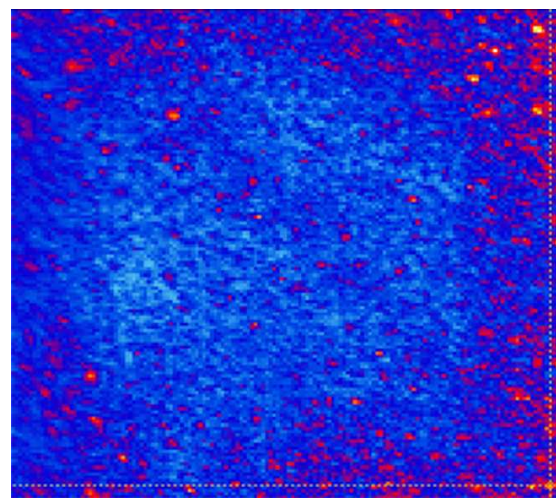

$100 \mu \mathrm{m}$

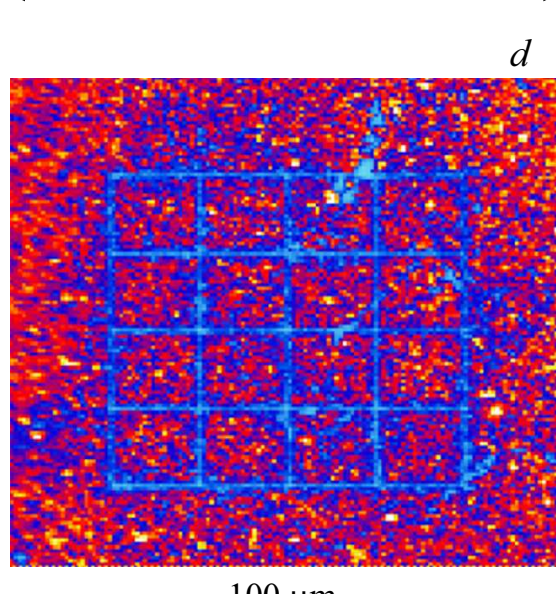

$100 \mu \mathrm{m}$ $b$

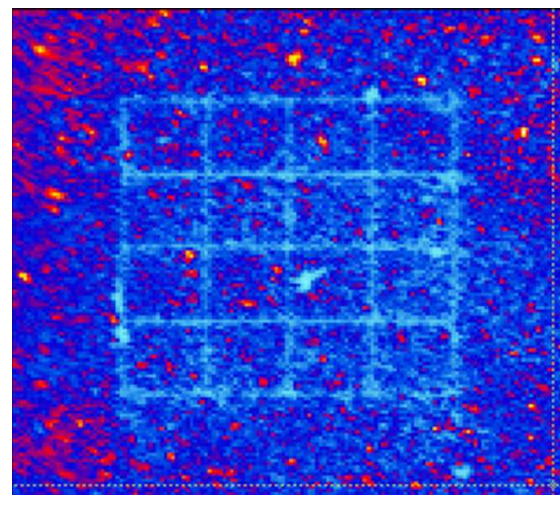

$100 \mu \mathrm{m}$

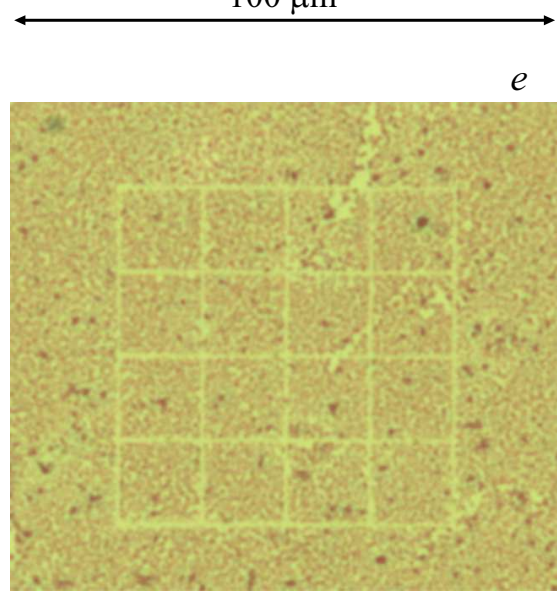

$e$

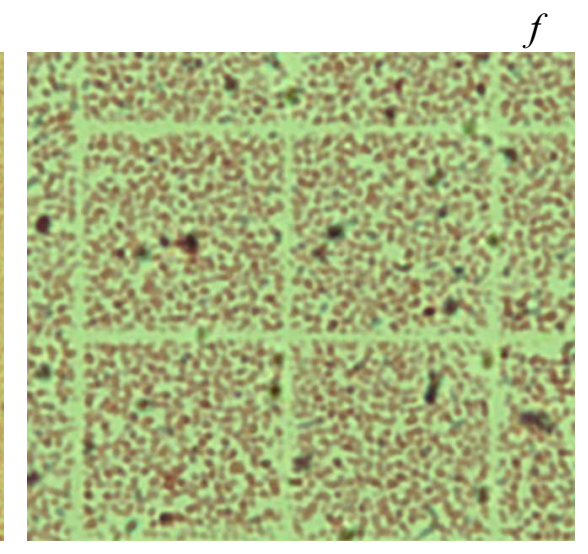

$15 \mu \mathrm{m}$

Рис. 3. Полученные путем сканирования лазерным лучом люминесцентные $(a-d)$ и снятые с помощью широкопольного оптического микроскопа $(e, f)$ изображения двумерных решеток с размером ячейки $15 \mu \mathrm{m}$, записанных при следующих парах параметров „мощность-скорость“: (a) $1 \mathrm{~mW}-5 \mu \mathrm{m} / \mathrm{s} ;$ (b) $5 \mathrm{~mW}-5 \mu \mathrm{m} / \mathrm{s} ;$ (c) $5 \mathrm{~mW}-10 \mu \mathrm{m} / \mathrm{s} ;$ (d) $10 \mathrm{~mW}-35 \mu \mathrm{m} / \mathrm{s}$; $(e, f) 10 \mathrm{~mW}-35 \mu \mathrm{m} / \mathrm{s}$.

\section{Вывод}

В ходе эксперимента подтверждена возможность записи 2D решеток в пленке РММА с субмикронными кристаллами DAST. Следовательно, можно записать ФКС структуру с необходимым периодом. При прямой лазерной записи с мощностью $10 \mathrm{~mW}$ и скоростью $35 \mu \mathrm{m} / \mathrm{s}$ была получена четко выраженная линия шириной менее $1 \mu \mathrm{m}$. Процесс записи не приводит к видимым разрушениям слоя и основан, вероятно, на полиморфных превращениях наночастиц.

Поскольку в работе использовался лазер, длина волны которого находится вблизи пика поглощения материала, то данная установка позволяет записывать только $2 \mathrm{D}$ изображения. Однако если применить инфракрасный лазер, который позволит использовать двухфотонное поглощение, можно рассчитывать на возможность записывать в аналогичном материале и $3 \mathrm{D}$ изображения, такие как 3D ФКС структуры.

\section{Финансирование работы}

Работа выполнена при поддержке Российского фонда фундаментальных исследований РФФИ (проект № 18-52-16014).

\section{Конфликт интересов}

Авторы заявляют, что у них нет конфликта интересов.

\section{Список литературы}

[1] Broderick N.G.R., Bratfalean R.T., Monro T.M., Richardson D.J., de Sterke C.M. // J. Opt. Soc. Am. B. 2002. V. 19. P. 2263.

[2] Saltiel S., Kivshar Y.S. // Opt. Lett. 2000 V. 25. P. 1204.

[3] Vodopyanov K.L., Levi O., Kuo P.S., Pinguet T.J., Harris J.S., Fejer M.M., Gerard B., Becouarn L., Lallier E. // Opt. Lett. 2004. V. 29. P. 1912.

[4] Imeshev G., Galvanauskas A., Harter D., Arbore M.A., Proctor M., Fejer M.M. // Opt. Lett. 1998. V. 23. P. 864. 
[5] Ellenbogen T., Voloch-Bloch N., Ganany-Padowicz A., Arie A. // Nat. Photon. 2009. V. 3. P. 395.

[6] Shapira A., Shiloh R., Juwiler I., Arie A. // Opt. Lett. 2012. V. 37. P. 2136.

[7] Meier U., Bosch M., Bosshard C., Gunter P. // Synthetic Metals. 2000. V. 109. P. 19-22.

[8] Forrest S., Burrows P., Stroustrup A., Strickland D., Ban V.S. // Appl. Phys. 1996. V. 68. P. 1326-1328.

[9] Pogosian T., Lai N.D. // Phys. Rev. A. 2016. V. 94. P. 063821.

[10] Li Q., Do M.T., Ledoux-Rak I., Lai N.D. // Opt. Lett. 2013. V. 38. P. 4640.

[11] Burunkova J.A., Denisyuk I.Y., Fokina M.I. // Mol. Cryst. Liq. Cryst. 2014. V. 589(1). P. 178.

[12] Thomas T., Ramaclus J.V., Mena F.P., Mosquera E., Sagayaraj P., Michael E.A. // Cryst. Eng.Commun. 2015. V. 17. P. 1989. 